\title{
Noise in resistively shunted Josephson junctions
}

\author{
F. T. Brandt t* and J. Frenke I \\ Instituto de Física, Universidade de São Paulo, \\ Rua do Matão, Travessa R, 187, \\ São Paulo, SP 05508-090, Brazil \\ J. C. Taylon \\ DAMTP, Centre for Mathematical Sciences, University of Cambridge, \\ Wilberforce Road, Cambridge, CB3 OWA, United Kingdom
}

\begin{abstract}
We investigate the dynamics of a resistively shunted Josephson junction. We compute the Josephson frequency and the generalized impedances for a variety of the parameters, particularly with relevance to predicting the measurable effects of zero-temperature current noise in the resistor.

PACS numbers: 05.40.-a,05.10.Gg,42.50.Lc,74.50.+r
\end{abstract}

\section{INTRODUCTION}

An experiment reported in 1981 [1] measured quantum noise in a resistively shunted Josephson junction. The high frequency noise (both thermal and quantum) in the resistor is mixed down to measurable frequencies by the non-linearity of the Josephson circuit. This experiment raised some important questions of principle. Quantum noise is not directly measurable, but it may have measurable effects in non-equilibrium situations [2]. The experiment has, controversially, been related to the question of "dark energy" 3]. The interpretation of the experiment involves a quantum Langevin equation (Eq. (1.4) below), and the status of this equation has been critically analyzed by one of us [4]. The existence of such an equation has been demonstrated but only in a simple independent-oscillator model [5].

In the experiment [1], a resistor $R$ is put in parallel with a Josephson junction at temperature $T$. The critical current for the junction is $I_{0}$ and a bias current $I>I_{0}$ is applied. The voltage across the junction is $V$, and noise fluctuations in this voltage are measured. The junction has capacitance $C$. Four different junctions were used with slightly different values of the parameters. As an example, for junction $2, C=0.8 \mathrm{pF}, I_{0}=0.5 \mathrm{~mA}, I=1$ $\mathrm{mA}, R=0.6 \Omega$, and one temperature for which details are given was $T=4.2 \mathrm{~K}$. The noise is measured at frequency $183 \mathrm{kHz}$.

The theory of the Josephson circuit is particularly simple if the capacitance $C$ can be neglected. In [1] a simple assumption is made about the dependence on $C$. One of the motivations for this paper is to test the validity of this assumption. We find significant deviations for some values of the voltage. Our results should be useful if experiments of a similar kind are performed in the future.

Our purpose here is to study the solutions of this

\footnotetext{
*Electronic address: fbrandt@usp.br

$\dagger$ Electronic address: jfrenkel@fma.if.usp.br

${ }^{\ddagger}$ Electronic address: J.C.Taylor@damtp.cam.ac.uk
}

Langevin equation, and to derive the Josephson frequency and the generalized impedances and hence predict the voltage noise strength. In the interpretation of the experiment [1], certain approximations were made, and we examine the validity of these approximations. We use two forms of perturbation theory, and also numerical methods.

A mechanical model for the dynamics of the Langevin equation (equation (1.4) below) has been studied experimentally and theoretically in [6].

The fundamental theoretical input [7] is the quantum version of the Nyquist fluctuation-dissipation theorem (originally derived in [8]). In the present context, it relates the current noise in the resistor at temperature $T$ to the conductance $1 / R$, and states

$$
\frac{1}{2}\left[S_{I}(\Omega)+S_{I}(-\Omega)\right]=\left(\frac{2 \hbar \Omega}{R}\right) \operatorname{coth}\left[\frac{\hbar \Omega}{2 T}\right]
$$

where

$$
S_{I}(\Omega) \delta\left(\Omega-\Omega^{\prime}\right)=\left\langle I_{n}(\Omega) I_{n}\left(-\Omega^{\prime}\right)\right\rangle
$$

$I_{n}(\Omega)$ being the Fourier transform of the current noise $I_{n}(t)$ in the resistor, and the expectation value $\langle\ldots\rangle$ is with respect to a thermal distribution at temperature $T$ (where we use units such that Boltzmann's constant is $1)$.

The right hand side of (1.1) has the finite limit

$$
\frac{2 \hbar \Omega}{R}
$$

as $T \rightarrow 0$, and this offers the possibility to measure quantum noise. For this it is essential to have the symmetrized version of $S_{I}$; the unsymmetrized $S_{I}(\Omega)$ itself has a vanishing low-temperature limit for $\Omega>0$. This reflects the impossibility of getting energy directly out of the vacuum. It is, of course, essential that $I_{n}$ is a quantum, non-commuting, operator.

The right hand side of (1.1) contains the distribution function for a quantum oscillator of frequency $\Omega$ at temperature $T$. However, as emphasized in [9], this by no 
means implies that the resistor contains oscillators, or even that the system is bosonic.

The second theoretical input is the assumed quantum Langevin equation, connecting the voltage and voltage noise to the current and current noise in the shunted Josephson circuit:

$$
\left(\frac{\hbar C}{2 e}\right) \ddot{\theta}+\left(\frac{\hbar}{2 e R}\right) \dot{\theta}+I_{0} \sin \theta=I+I_{n}
$$

where $\theta$ is the Josephson phase (including noise) and $C$ is the capacitance of the junction. Since $I_{n}$ is a quantum operator, $\theta$ must be also. The status of this equation is less secure than that of (1.1) (see [4]). The voltage $V$ across the junction is given by

$$
V(t)=\frac{\hbar}{2 e} \dot{\theta}
$$

We will always assume that $I>I_{0}$. Then, in the absence of the noise $I_{n}$, the solutions $\theta_{c}(t-\hat{t})$ of (1.4) settle down, after the decay of transients, to a periodic dependence on time, with frequency $\Omega_{J}=2 \pi / T_{J}$, so that

$$
\theta_{c}\left(t+T_{J}-\hat{t}\right)=\theta_{c}(t-\hat{t})+2 \pi
$$

for all $t$. The arbitrary constant $\hat{t}$ is included in order to make explicit the infinite set of solution depending on the initial condition (although (1.4) is a second order equation, the initial value of $\dot{\theta}$ is not an independent initial condition for the steady periodic solution).

On the right of (1.4), the current noise $I_{n}$ is certainly a quantum operator, but $I$ is presumably a classical quantity. Since $I_{n}$ is small, we can write

$$
\theta=\theta_{c}+\theta_{n}
$$

where $\theta_{c}$ is the (classical) solution and $\theta_{n}$ is the small quantum noise. Then the latter obeys, to a good approximation, the linear quantum equation

$$
\left(\frac{\hbar C}{2 e}\right) \ddot{\theta}_{n}+\left(\frac{\hbar}{2 e R}\right) \dot{\theta}_{n}+I_{0}\left(\cos \theta_{c}\right) \theta_{n}=I_{n}
$$

in which $\cos \theta_{c}$ is a $c$-number coefficient.

Because of (1.6), there is a Fourier series (we use $j$ for $\sqrt{-1})$

$$
\begin{aligned}
\cos \theta_{c}(t-\hat{t}) & =\sum_{k} g_{k} \exp \left(j k \Omega_{J} t\right) \exp \left(-j k \hat{t} \Omega_{J}\right) \\
& \equiv \sum_{k} g_{k}(\hat{t}) \exp \left(j k \Omega_{J} t\right)
\end{aligned}
$$

with $k$ an integer and $g_{k}^{*}=g_{-k}$. We define a hybrid Fourier integral/series by

$$
I_{n}(t)=\sum_{k} \int_{0}^{\Omega_{J}} d \Omega I_{n k}(\Omega) \exp \left[j t\left(\Omega+k \Omega_{J}\right)\right]
$$

and similarly for $\theta$ and $V=(\hbar / 2 e) d \theta / d t$. Then the transform of (1.8) is

$$
\begin{aligned}
& \sum_{k^{\prime}}\left[F_{k}(\Omega) \delta_{k, k^{\prime}}+I_{0} g_{k-k^{\prime}}(\hat{t})\right] \theta_{n k^{\prime}} \\
& \equiv \sum_{k^{\prime}} X_{k, k^{\prime}}(\Omega) \theta_{n k^{\prime}}(\Omega)=I_{n k}(\Omega) .
\end{aligned}
$$

where

$$
\begin{aligned}
F_{k}(\Omega) & =-\left(\frac{\hbar C}{2 e}\right)\left(\Omega+k \Omega_{J}\right)^{2} \\
& +j\left(\frac{\hbar}{2 e R}\right)\left(\Omega+k \Omega_{J}\right)
\end{aligned}
$$

Eq. (1.11) has solution

$$
\theta_{n k}(\Omega)=\sum_{k^{\prime}} Y_{k k^{\prime}}(\Omega) I_{n k}(\Omega)
$$

where the matrix $Y$ is the inverse of $X$ (for each $\Omega$ ).

We assume that there is a non-zero limit

$$
\lim _{\Omega \rightarrow 0}\left[j \Omega Y_{0, k}(\Omega)\right] \equiv \frac{2 e}{\hbar} Z_{k}
$$

so that (by (1.5))

$$
V_{n 0}(0)=\sum_{k} Z_{k} I_{n k}(0)
$$

This is certainly true for $C=0$ [10], and also consistent with our conclusions in the remainder of this paper.

We have not made explicit the $\hat{t}$ dependence of $X, Y$ and $Z$, but it follows from (1.9), (1.10), (1.13) and (1.14) that $Z$ is proportional to $\exp \left(-j k \hat{t} \Omega_{J}\right)$.

We define a periodic function $\tilde{I}_{n}(t)$ associated with $I_{n}$ by

$$
\tilde{I}_{n}(t)=\sum_{k} I_{n k}(\Omega=0) \exp \left(j t k \Omega_{J}\right)
$$

and similarly for $\tilde{V}_{n}$ Then, for the purpose of computing the $Z_{k}$ defined in (1.14) it is sufficient to use $\tilde{I}_{n}$ and $\tilde{V}_{n}$.

The basic problem is to obtain from (1.1) and (1.8) information about the voltage noise (using (1.5)). One approach to this problem [7, 11] is to simulate the current noise numerically, but it is not clear how to do this consistently with the frequency dependence in (1.1). Also, the noise is supposed to be a quantum operator. Our method is indirect. We first obtain the impedances $Z_{k}$ defined in (1.15). Since these are assumed to be classical quantities (this is the consequence of the assumed existence of a quantum Langevin equation with classical coefficients in it), to find them it is sufficient to take the noise in (1.8) to be classical. Having found the impedances $Z_{k}$, we can use (1.15) (with quantum noise) together with (1.1) to determine the strength of the (quantum) voltage noise. An advantage of this method is that (1.1) is exactly respected.

Thus, the purpose of this paper is to study the solutions of equation (1.4) and (1.8), and the calculation of 
$\Omega_{J}$ and $Z_{k}$. This enables us to check the validity of some of the approximations made in [1]. In section [II], we use perturbation theory for small $C$. This turns out to be a series in $C^{2}$, and we are able to calculate the $O\left(C^{2}\right)$ contribution to $\Omega_{J}$, and to $Z_{k}$ but for $k>1$ only. In section V] we use perturbation theory in $1 / I$. Again, there are only even terms, but we calculate $O\left(1 / I^{2}\right)$ contributions to $\Omega_{J}$ and to $Z_{k}$. But this approximation is not useful for the values of $I$ in the experiments. In section VI we give results of numerical calculations of $\Omega_{J}, R_{D}$ and $Z_{1}, Z_{2}$, for several values of $\beta$ and $i$ (dimensionless parameters defined in (2.1) and (2.5) below). We differ from some other numerical work [7, 11] in that we do not attempt to simulate the actual noise (which should be consistent with (1.1) ), but just determine the $Z_{k}$ factors. Our conclusions are given in graphs, which may be useful in the interpretation of experiments.

One particular conclusion is that, in the range $0<\beta<$ 0.2 (with $\beta$ defined as in (2.1) below) $Z_{1}$ decreases and $R_{D}$ increases. In Table II we show by what factors the values of $Z_{1}^{2}$ and of $\left(Z_{1} / R_{D}\right)^{2}$ are predicted to change between $\beta=0$ and $\beta=0.38$. As we shall discuss, these ratios are new corrections to the predicted voltage noise.

We conclude that the apparent quantitative agreement between theory and observation in [1] may not be as good as it appears. Certainly, our calculations should be relevant to any future repetition of this type of experiment. For another possible applications of equation (1.4), see Ref. [12].

\section{NOTATION AND A SIMPLE SOLUTION}

It is useful to define the dimensionless variables

$$
\begin{gathered}
i=\frac{I}{I_{0}}, v=\frac{V}{I_{0} R}, \tau=\frac{2 e R I_{0}}{\hbar} t \\
\beta=\frac{2 e R^{2} C I_{0}}{\hbar}, \omega=\frac{\hbar}{2 e R I_{0}} \Omega, z_{k}=\frac{Z_{k}}{R} .
\end{gathered}
$$

In terms of these variables, and denoting $d \theta / d \tau$ by $\theta^{\prime}$, equation (1.4) becomes in the absence of the current noise

$$
\beta \theta^{\prime \prime}+\theta^{\prime}+\sin \theta=i .
$$

In the special case when $\beta$ is negligible, this equation is easily soluble. A particularly convenient form of a solution is

$$
\begin{aligned}
\sin \theta_{0} & =\frac{1+i \sin \left(\omega_{J 0} \tau\right)}{i+\sin \left(\omega_{J 0} \tau\right)} \\
\cos \theta_{0} & =\frac{\omega_{J 0} \cos \left(\omega_{J 0} \tau\right)}{i+\sin \left(\omega_{J 0} \tau\right)} \\
v_{0}=\frac{d \theta_{0}}{d \tau} & =\frac{\omega_{J 0}^{2}}{i+\sin \left(\omega_{J 0} \tau\right)}
\end{aligned}
$$

where in general $\omega_{J}$ is the natural frequency of the solutions of (2.2) (when transient decaying terms have died out), and $\omega_{J 0}$ is the value in this approximation $(\beta \simeq 0)$ :

$$
\omega_{J 0}=\sqrt{i^{2}-1}
$$

Similarly, $\theta_{0}$ denotes the solution of (2.2) when $\beta=0$. In this case, equation (1.14) gives only three non-zero values of $Z_{k}$, with $k=0, \pm 1$.

The dynamic resistance is defined to be

$$
R_{D}=\frac{d \bar{V}}{d I}, r_{D} \equiv z_{0}=\frac{d \bar{v}}{d i}
$$

where $\bar{V}$ denotes the time-average over one cycle. In this approximation $(\beta=0)$

$$
\bar{v}=\frac{2 \pi}{\tau_{J 0}}=\omega_{J 0},
$$

so, from 2.4

$$
r_{D 0}=\frac{i}{\omega_{J 0}}
$$

Equation (1.15) gives

$$
\begin{aligned}
\left\langle V_{n 0}(0) V_{0 n}^{*}(0)\right\rangle_{\hat{t}} & =\sum_{k, k^{\prime}} \exp \left[-j \hat{t} \Omega_{J}\left(k-k^{\prime}\right)\right] \\
& \times Z_{k} Z_{k^{\prime}}^{*}\left\langle I_{n k}(0) I_{n k^{\prime}}^{*}(0)\right\rangle
\end{aligned}
$$

where we have made explicit the $\hat{t}$ dependence in (1.14) inherited from (1.9). We now average (2.8) over $\hat{t}$ through one period $T_{J}$ and use (1.2) to get

$$
\begin{aligned}
S_{V}(0) & =\left\langle V_{n 0}(0) V_{n 0}^{*}(0)\right\rangle \\
& \equiv \frac{1}{T_{J}} \int_{0}^{T_{J}} d \hat{t}\left\langle V_{n 0}(0) V_{n 0}^{*}(0)\right\rangle_{\hat{t}} \\
& =\sum_{k}\left|Z_{k}\right|^{2} S_{I}\left(k \Omega_{J}\right) .
\end{aligned}
$$

Since $Z_{k}^{*}=Z_{-k}$, the right hand side of this equation automatically contains the symmetrized products of currents, as in equation (1.1). Finally using (1.1) with $\Omega=k \Omega_{J}$, (2.9) gives the required prediction for the low frequency voltage noise $S_{V}(0)$. 


$$
S_{V}(0)=2 \sum_{k}\left|Z_{k}\right|^{2}\left(\frac{\hbar k \Omega_{J}}{R}\right) \operatorname{coth}\left[\frac{\hbar k \Omega_{J}}{2 T}\right]=4 e R^{2} I_{0}\left[\frac{\left|z_{0}\right|^{2}}{p}+\sum_{k \geq 1} k\left|z_{k}\right|^{2} \omega_{J} \operatorname{coth}\left(p k \omega_{J}\right)\right],
$$

where we have used (2.1) and $p=e R I_{0} / T$ (and $p$ is close to 1 in the experiment. 1]).

For the special case $C \simeq 0$, we have

$$
Z_{0}=\frac{R I}{\sqrt{I^{2}-I_{0}^{2} \mid}}, \quad\left|Z_{ \pm 1}\right|=\frac{R I_{0}}{2 \sqrt{I^{2}-I_{0}^{2}}}
$$

and all other $Z_{k}=0$. Then (2.10) gives

$$
\begin{aligned}
S_{V}(0) & =\frac{4 T R I^{2}}{I^{2}-I_{0}^{2}}+\frac{e R^{2} I_{0}^{2}}{\sqrt{I^{2}-I_{0}^{2}}} \\
& \times \operatorname{coth}\left[\frac{e R \sqrt{I^{2}-I_{0}^{2}}}{2 T}\right] .
\end{aligned}
$$

Using (2.1), (2.4), (2.5) and (2.6), this may alternatively be written

$$
\frac{S_{V}(0)}{R_{D}^{2}}=\frac{4 T}{R}+\frac{2 e \bar{V} I_{0}^{2}}{R I^{2}} \operatorname{coth}\left[\frac{e \bar{V}}{T}\right] .
$$

It has been suggested [1] that in this form, if observed values of $\bar{V}$ and $R_{D}$ are used, it may also be a good approximation for non-zero $\beta$ (defined in (2.1)). One purpose of this paper is to test this approximation. We note from (2.10) that the quantity $S_{V}(0) / R_{D}^{2}$ in (2.13) depends upon the magnitudes of the ratios $\left(Z_{k} / R_{D}\right)^{2}$.

\section{PERTURBATION THEORY FOR SMALL CAPACITANCE}

This section is about the calculation of the Josephson frequency; it is not concerned with noise, so all variables are classical ones.

Since $\beta$ in equation (2.2) has modest values in the experiments [1], one might expect perturbation theory in $\beta$ to be useful. This is especially so as the series turns out to be an expansion in $\beta^{2}$. This property follows because (2.2) is invariant under

$$
\tau \rightarrow-\tau, \beta \rightarrow-\beta, \quad \theta \rightarrow \pi-\theta, \quad i \rightarrow+i .
$$

To construct the perturbation series, we expand

$$
\theta=\theta_{0}+\beta \theta_{1}+\beta^{2} \theta_{2}+\ldots,
$$

and

$$
\tau_{J}=\tau_{J 0}+\beta \tau_{J 1}+\beta^{2} \tau_{J 2}+\ldots,
$$

where $\tau=2 \pi / \omega_{J}$ is the Josephson period and $\tau_{J 0}$ is given by (2.4). Inserting (3.2) into (2.2),

$$
\theta_{1}^{\prime}+\left(\cos \theta_{0}\right) \theta_{1}=-\theta_{0}^{\prime \prime},
$$

$$
\theta_{2}^{\prime}+\left(\cos \theta_{0}\right) \theta_{2}=-\theta_{1}^{\prime \prime}+\frac{\theta_{1}^{2}}{2} \sin \theta_{0}
$$

where prime denotes differentiation with respect to $\tau$.

Equation (3.4) gives, using (2.3),

$$
\left[i+\sin \left(\omega_{J 0} \tau\right) \theta_{1}\right]^{\prime}=\frac{\omega_{J 0}^{3} \cos \left(\omega_{J 0} \tau\right)}{i+\sin \left(\omega_{J 0} \tau\right)} .
$$

Hence

$$
\theta_{1}=\frac{\omega_{J 0}^{2} \ln \left[1+\sin \left(\omega_{J 0} \tau\right) / i\right]}{i+\sin \left(\omega_{J 0} \tau\right)}
$$

where we have chosen the initial value $\theta(0)=1 / i$. This solution has the same frequency $\omega_{J 0}$ as $\theta_{0}$; so $\tau_{1}=0$ in (3.3). This is in accordance with our remark above that the expansion is series in $\beta^{2}$.

From the definition of $T$, for any $\tau$, to the requisite order,

$$
\begin{aligned}
2 \pi & =\theta\left(\tau+\tau_{J}\right)-\theta(\tau) \\
& =\theta_{0}\left(\tau+\tau_{J}\right)-\theta_{0}(\tau) \\
& +\beta^{2}\left[\theta_{2}\left(\tau+\tau_{J 0}\right)-\theta(\tau)\right],
\end{aligned}
$$

giving

$$
\tau_{J 2}=-\left[\theta_{2}\left(\tau+\tau_{J 0}\right)-\theta_{2}(\tau)\right] / \theta_{J 0}^{\prime}(\tau) .
$$

(To be consistent, the right hand side of this equation must turn out to be independent of $\tau$.)

Then, from (3.5), (3.7) and (3.9), and using (2.3),

$$
\begin{aligned}
\tau_{J 2} & =\frac{1}{\omega_{J 0}{ }^{2}} \int_{\tau}^{\tau+\tau_{J 0}} d \tau^{\prime}\left[i+\sin \left(\omega_{J 0} \tau^{\prime}\right)\right] \\
& \times\left[\theta_{1}^{\prime \prime}\left(\tau^{\prime}\right)-\frac{1}{2}\left(\sin \theta_{0}\right) \theta_{1}\left(\tau^{\prime}\right)^{2}\right] \\
& =-\omega_{J 0}^{2} \int_{\tau}^{\tau+\tau_{J 0}} d \tau^{\prime} \\
& \times\left[\sin \left(\omega_{J 0} \tau^{\prime}\right) \ln \left(1+\frac{\sin \left(\omega_{J 0} \tau^{\prime}\right)}{i}\right)\right. \\
& +\frac{1+i \sin \left(\omega_{J 0} \tau^{\prime}\right)}{\left(i+\sin \left(\omega_{J 0} \tau^{\prime}\right)\right)^{2}} \\
& \left.\times \ln ^{2}\left(1+\frac{\sin \left(\omega_{J 0} \tau^{\prime}\right)}{i}\right)\right]
\end{aligned}
$$

where we have integrated by parts the $\theta_{1}^{\prime \prime}$ term in the first line. It turns out that all the integrals arising in 3.10 which contain a power of $\ln \left(1+\sin \left(\omega_{J 0} \tau\right) / i\right)$ can be done 
by integration by parts:

$$
\begin{aligned}
\tau_{J 2} & =-\omega_{J 0} \int_{\tau}^{\tau+\tau_{J 0}} d \tau^{\prime} \\
& \times\left[\frac{\omega_{J 0} \cos ^{2}\left(\omega_{J 0} \tau^{\prime}\right)}{\left(i+\sin \left(\omega_{J 0} \tau^{\prime}\right)\right)^{2}}-\frac{d H}{d \tau^{\prime}}\right]
\end{aligned}
$$

Where

$$
\begin{aligned}
H(\tau) & =\frac{\cos \left(\omega_{J 0} \tau\right)}{i+\sin \left(\omega_{J 0} \tau\right)}\left[\frac{1}{2} \ln ^{2}\left(1+\frac{\sin \omega_{J 0} \tau}{i}\right)\right. \\
& \left.-\ln \left(1+\frac{\sin \left(\omega_{J 0} \tau\right.}{i}\right)+1\right] .
\end{aligned}
$$

It is now a simple matter to complete the integration and find

$$
\begin{aligned}
\tau_{J 2} & =-\omega_{J 0}^{2} \int_{\tau}^{\tau+\tau_{J 0}} d \tau^{\prime} \frac{\cos ^{2}\left(\omega_{J 0} \tau^{\prime}\right)}{\left(i+\sin \left(\omega_{J 0} \tau^{\prime}\right)\right)^{2}} \\
& =-2 \pi\left(i-\omega_{J 0}\right) .
\end{aligned}
$$

Thus the Josephson period to second order is

$$
\tau_{J}=\tau_{J 0}\left[1-\beta^{2} \omega_{J 0}\left(i-\omega_{J 0}\right)\right]
$$

and the frequency is

$$
\omega_{J}=\omega_{J 0}\left[\left(1+\beta^{2} \omega_{J 0}\left(i-\omega_{J 0}\right)\right] .\right.
$$

From (2.6), in this approximation the dynamical resistance is

$$
r_{D}=z_{0}=\frac{i}{\omega_{J 0}}+\beta^{2}\left(i-\omega_{J 0}\right)\left(2 i-\omega_{J 0}\right) .
$$

Figures 2 and 3 show a comparison of (3.15) and (3.16) with numerical results.

\section{PERTURBATION THEORY FOR IMPEDANCE OF HIGHER HARMONICS}

In this section, we use perturbation theory in $\beta$ to study the impedances $z_{k}$. Because $z_{1}$ has a contribution (see (2.11) ) to zeroth order in $\beta$,

$$
\left|z_{1}\right|^{2}=\left|z_{10}+\beta z_{11}+\beta^{2} z_{12}+\ldots\right|^{2}
$$

and we need the second order term because of its interference with the zeroth order one; so first order perturbation theory on its own has no physical significance. We have found second order perturbation theory prohibitively complicated. But for $k>1$, there is no zeroth order contribution and so first order perturbation theory is relevant by itself, and this we now study.

Since our aim is to calculate the impedances $z_{k}$ (which are classical quantities), it is sufficient to introduce, instead of the quantum noise $i_{n}$, a classical driving force at the Josephson frequency $\omega_{J}$ into (2.2). To our approximation, it is sufficient to use Eq. (2.4). Thus we use the equation

$$
\beta \theta^{\prime \prime}+\theta^{\prime}+\sin \theta=i+a_{k} \exp \left(j k \omega_{J 0} \tau\right)
$$

where $a_{k}$ is complex and infinitesimal. We expand

$$
\theta=\theta_{0}+a_{k} \theta_{a_{k}}+b \theta_{1}+b a_{k} \theta_{1 a_{k}}+\ldots
$$

Then

$$
\theta_{a_{k}}^{\prime}+\theta_{a_{k}} \cos \theta_{0}=\exp \left(i k \omega_{J 0}\right)
$$

so

$$
\begin{gathered}
{\left[\left(i+\sin \left(\omega_{J 0} \tau\right)\right)\right] \theta_{a_{k}}=F} \\
\equiv \int_{\tau_{0}}^{\tau+\tau_{0}} d \tau^{\prime}\left[\left(i+\sin \left(\omega_{J 0} \tau^{\prime}\right)\right] \exp \left(j k \omega_{J 0} \tau^{\prime}\right) .\right.
\end{gathered}
$$

$\theta_{1}$ is given in (3.7).

To the next order,

$$
\theta_{1 a_{k}}^{\prime}+\left(\cos \theta_{0}\right) \theta_{1 a_{k}}=\left(\sin \theta_{0}\right) \theta_{a_{k}} \theta_{1}-\theta_{a_{k}}^{\prime \prime}
$$

So, using (2.3) and (4.5),

$$
\begin{gathered}
\frac{d}{d \tau}\left[\left(i+\sin \left(\omega_{J 0} t\right)\right) \theta_{1 a_{k}}\right]= \\
\omega_{J 0}^{2} \frac{1+i \sin \left(\omega_{J 0} \tau\right)}{i+\sin \left(\omega_{J 0} t \tau\right)} \ln \left[1+\frac{\sin \left(\omega_{J 0} \tau\right)}{i}\right] \theta_{a_{k}} \\
-\left[i+\sin \left(\omega_{J 0} \tau\right)\right] \theta_{a_{k}}^{\prime \prime} .
\end{gathered}
$$

We want to determine the period of $\theta$ in (4.2). Let this period be

$$
\tau_{J}=\tau_{J 0}+b a_{k} \tau_{1 a_{k}}+\ldots
$$

then

$$
\begin{aligned}
2 \pi & =\theta_{0}\left(\tau+\tau_{J 0}+b a_{k} \tau_{1 a_{k}}\right)-\theta_{0}(\tau) \\
& +b a_{k}\left[\theta_{1 a_{k}}\left(\tau+\tau_{J 0}\right)-\theta_{1 a_{k}}(\tau)\right]
\end{aligned}
$$

where $\tau$ is arbitrary, so

$$
\tau_{1 a_{k}} \theta_{0}^{\prime}(\tau)=-\left[\theta_{1 a_{k}}\left(\tau+\tau_{J 0}\right)-\theta_{1 a_{k}}(\tau)\right] .
$$

It follows from (1.4) and (4.6) that

$$
\begin{aligned}
\omega_{J 0}^{2} \tau_{1 a_{k}} & =-\int_{\tau}^{\tau+\tau_{J 0}} d \tau^{\prime}\left[\omega_{J 0}^{2} \frac{1+i \sin \left(\omega_{J 0} \tau^{\prime}\right)}{\left(i+\sin \left(\omega_{J 0} \tau^{\prime}\right)\right)^{2}}\right. \\
& \times \ln \left[1+\frac{\sin \omega_{J 0} \tau}{i}\right] \theta_{a_{k}}\left(\tau^{\prime}\right) \\
& \left.-\left(i+\sin \left(\omega_{J 0} \tau^{\prime}\right)\right) \theta_{a_{k}}^{\prime \prime}\left(\tau^{\prime}\right)\right]
\end{aligned}
$$

Integrating by parts and using the periodicity of the integrand, the second term in the square bracket in 4.10) is converted to

$$
\omega_{J 0}^{2} \sin \left(\omega_{J 0} \tau^{\prime}\right) \theta_{1 a_{k}}
$$


Then, using (4.4), (4.10) gives

$$
\begin{aligned}
\tau_{1 a_{k}} & =-\frac{1}{\omega_{J 0}} \int_{\tau}^{\tau+\tau_{J 0}} d t^{\prime}\left\{F^{\prime}\left(\tau^{\prime}\right) \frac{\cos \left(\omega_{J 0} \tau^{\prime}\right)}{\sin \left(\omega_{J 0} \tau^{\prime}\right)}\right. \\
& \left.\times\left[1+\ln \left(1+\frac{\sin \left(\omega_{J 0} \tau^{\prime}\right)}{i}\right)\right]-G^{\prime}\left(\tau^{\prime}\right)\right\}
\end{aligned}
$$

where

$$
\begin{aligned}
G(\tau) & =\frac{\cos \left(\omega_{J 0} \tau\right)}{i+\sin \left(\omega_{J 0} \tau\right)} F(\tau) \\
& \times\left[1+\ln \left(1+\frac{\sin \left(\omega_{J 0} \tau\right)}{i}\right)\right]
\end{aligned}
$$

Again, because of the periodicity, the contribution from the $G^{\prime}$ term in (4.12) is zero. Finally, inserting the value of $F^{\prime}$ from (4.4) and omitting an integral of a differential of a periodic function, we get

$$
\begin{aligned}
\tau_{1 a_{k}} & =-\frac{1}{\omega_{J 0}} \int_{\tau}^{\tau+\tau_{J 0}} d \tau^{\prime} \cos \left(\omega_{J 0} t^{\prime}\right) \\
& \times \ln \left[1+\frac{\sin \left(\omega_{J 0} \tau^{\prime}\right)}{i}\right] \exp \left(j k \omega_{J 0} \tau^{\prime}\right) .
\end{aligned}
$$

Because the integrand in (4.14) is periodic, we may replace the limits of integration by 0 and $\tau_{J 0}$ Integrating by parts again, it gives

$$
\begin{aligned}
\tau_{1 a_{k}} & =\frac{1}{2 \omega_{J 0}} \int_{0}^{\tau_{J 0}} d \tau^{\prime} \frac{\cos \left(\omega_{J 0} \tau^{\prime}\right)}{i+\sin \left(\omega_{J 0} \tau^{\prime}\right)} \\
& \times\left[\frac{\exp \left(j(k+1) \omega_{J 0} t \tau^{\prime}\right)}{k+1}+\frac{\exp \left(j(k-1) \omega_{J 0} \tau^{\prime}\right)}{k-1}\right] .
\end{aligned}
$$

The integral may now be done by the substitution $\zeta=$ $\exp \left(j \omega_{J 0} \tau^{\prime}\right)$ and integrating round the unit circle. There are poles at $\zeta=-j u,-j u^{-1}$ where

$$
u=i+\omega_{J 0}, \quad u^{-1}=i-\omega_{J 0},
$$

and $-j u^{-1}$ lies within the unit circle (taking $i>1$ for the moment). Remembering that

$$
z_{k}=\beta \omega_{1 a_{k}}=-\beta \omega_{J 0}^{2} \frac{\tau_{1 a_{k}}}{2 \pi},
$$

the result is

$$
z_{k}=(-j)^{k+1} \frac{\beta}{2}\left[\frac{u^{-k+1}}{k-1}-\frac{u^{-k-1}}{k+1}\right] .
$$

(The restriction $i \neq 1$ may now be relaxed.)

For low enough temperature, or high enough current $I$, when $p$ in (2.10) is considerably greater than 1, equation (2.10) for the voltage noise approximately contains the sum

$$
\sum_{k>1} k\left|z_{k}\right|^{2}
$$

which, in the small $\beta$ approximation (4.18), may be evaluated to give

$$
\beta^{2}\left[\frac{5}{16}-\frac{\omega_{J 0}^{2}}{4\left(i+\omega_{J 0}\right)^{2}}+\omega_{J 0}^{2} \ln \left(\frac{2 \omega_{J 0}}{i+\omega_{J 0}}\right)\right] .
$$

In the experiment [1], $k$ is of order 1 , but still (4.19) is a lower bound to the contributions from $k>1$ (since the coth factors are greater than 1 ).

\section{PERTURBATION THEORY FOR HIGH CURRENT}

In this section, we develop an expansion in powers of $1 / i=I_{0} / I$. This is algebraically simpler than the expansion in $\beta$, but is relevant only to a small range of $I$ in the experiments.

Because of the invariance of (2.2) under $i \rightarrow-i, \theta \rightarrow$ $-\theta$, the expansion is in powers of $1 / i^{2}$.

Define $s=i \tau$ and a function $\vartheta(s)=\theta(\tau)$. Then (4.1) becomes

$$
(i \beta) \vartheta^{\prime \prime}+\vartheta^{\prime}+\frac{1}{i} \sin \vartheta=1+\frac{a_{k}}{i} \exp (j k s),
$$

and we will be concerned only with $k=1$ or 2 in this section. In general the frequency of the last term in (5.1) ought to be $\omega_{J}$, but to our order it is sufficient to use the zeroth approximation $\omega_{J 0} \approx i$. Since the driving force is classical, $\theta$ is also classical in this section and in section VI.

Expand

$$
\begin{aligned}
\vartheta & =\vartheta_{0}+\frac{1}{i} \vartheta_{1}+\frac{1}{i^{2}} \vartheta_{2} \\
& +\frac{a_{1}}{i} \vartheta_{1 a_{1}}+\frac{a_{1}}{i^{2}} \vartheta_{2 a_{1}}+\frac{a_{2}}{i} \vartheta_{1 a_{2}}+\ldots
\end{aligned}
$$

with similar expansions of $\tau_{J}$ and $\omega_{J}$. (We will find that $\tau_{n a_{k}}=0$ for $n<k+1$.) We take $\vartheta_{0}=s$, being the solution without a transient decreasing exponential. Then

$$
(i \beta) \vartheta_{I}^{\prime \prime}+\vartheta_{1}^{\prime}=-\sin \vartheta_{0}=-\sin s
$$

$$
(i \beta) \vartheta_{1 a_{1}}^{\prime \prime}+\vartheta_{1 a_{1}}^{\prime}=\exp (j s)
$$

$$
(i \beta) \vartheta_{2}^{\prime \prime}+\vartheta_{2}^{\prime}=-\left(\cos \vartheta_{0}\right) \vartheta_{1}=-(\cos s) \vartheta_{1},
$$

$$
(i \beta) \vartheta_{2 a_{2}}^{\prime \prime}+\vartheta_{2 a_{2}}^{\prime}=-\left(\cos \vartheta_{0}\right) \vartheta_{1 a_{1}}=-(\cos s) \vartheta_{1 a_{1}} \text {. }
$$

The solution of (5.3) with no transient, and a convenient initial value, is

$$
\vartheta_{1}=\frac{1}{2}\left[\frac{\exp (j s)}{1+j g}+\frac{\exp (-j s)}{1-j g}\right]
$$

where

$$
g=i \beta .
$$


Since (5.7) is periodic with the same period as $\vartheta_{0}$, the period is not changed to first order, as expected.

The solution of (5.4) is

$$
\vartheta_{1 a_{1}}=\frac{-j \exp (j s)}{1+j g} .
$$

Again, the period is unaltered, and so there is no correction to $\bar{v}$ or to $z_{0}$ at this order.

Inserting (5.7) into (5.5),

$$
\begin{aligned}
(i \beta) \vartheta_{2}^{\prime \prime}+\vartheta_{2} & =-\frac{1}{4\left(1+g^{2}\right)} \\
& \times[2+(1-j g) \exp (2 j s) \\
& +(1+j g) \exp (-2 j s)] .
\end{aligned}
$$

so

$$
\vartheta_{2}(s+2 \pi)-\vartheta_{2}(s)=-\frac{\pi}{1+g^{2}} .
$$

It follows, in a similar manner to (4.8), the period at this order is

$$
\tau_{J 0}+\frac{1}{i^{2}} \tau_{J 2}=\frac{2 \pi}{i}\left[1+\frac{1}{2 i^{2}\left(1+g^{2}\right)}\right],
$$

and

$$
\begin{aligned}
\bar{v} & =\omega_{J}=i\left[1-\frac{1}{2 i^{2}\left(1+g^{2}\right)}\right], \\
r_{D} & =\frac{d \omega_{J}}{d i}=1+\frac{1+3 g^{2}}{2 i^{2}\left(1+g^{2}\right)^{2}},
\end{aligned}
$$

to this order. (For $\beta=0$, this is consistent with (2.4)).

Inserting (5.9) into (5.6), we find that

$$
\vartheta_{2 a_{2}}=-\frac{\exp (2 j s)}{[2(j-g)(2 j-4 g)]}-\frac{s}{[2(j-g)]},
$$

hence

$$
\vartheta_{2 a_{2}}(s+2 \pi)-\vartheta_{a 2}(s)=-\pi \frac{1}{(j-g)} .
$$

Therefore

$$
\tau_{2 a_{1}}=\pi \frac{i}{(j-g)},
$$

and

$$
z_{1}=-\frac{1}{2 \pi i^{2}} \tau_{2 a_{1}}=-\frac{1}{2 i(j-g)}, \quad\left|z_{1}\right|=\frac{1}{2 i \sqrt{1+g^{2}}} .
$$

Comparisons of (5.13) and (5.17) with numerical data are shown in Figs. 2, 3 and 4 The perturbation theory is in reasonable agreement for $i>2.0$.

We can use the expansion in $1 / i$ for $k=2$, but we must go to one order higher in $1 / i$ to find $z_{2}$. We use the $a_{2}$ terms in (5.1) and (5.2). The equations are

$$
\begin{aligned}
& g \vartheta_{1 a_{2}}^{\prime \prime}+\vartheta_{1 a_{2}}^{\prime}=\exp (2 j s), \\
& g \vartheta_{2 a_{2}}^{\prime \prime}+\vartheta_{2 a_{2}}^{\prime}=-(\cos s) \vartheta_{1 a_{2}}, \\
& g \vartheta_{3 a_{2}}^{\prime \prime}+\vartheta_{3 a_{2}}^{\prime}=-(\cos s) \vartheta_{2 a_{2}}+(\sin s) \vartheta_{1} \vartheta_{1 a_{2}} .
\end{aligned}
$$

Then

$$
\begin{aligned}
\vartheta_{1 a_{2}} & =\frac{\exp (2 j s)}{2 j-4 g} \\
\vartheta_{2 a_{2}} & =-\frac{\exp (j s)}{[2(j-g)(2 j-4 g)]} \\
& -\frac{\exp (3 j s)}{[2(9 g-3 j)(4 g-2 j)]},
\end{aligned}
$$

where the second term will turn out to be irrelevant,

Putting (5.19) into the last of (5.18) and using (5.7),

$$
\begin{aligned}
g \vartheta_{3 a_{2}}^{\prime \prime}+\vartheta_{3 a_{2}}^{\prime} & =\frac{1}{[4(j-g)(2 j-4 g)]} \\
& -\frac{1}{[4(2 j-4 g)(j+g)]}+\ldots
\end{aligned}
$$

where the omitted terms are periodic and irrelevant. Hence

$$
\vartheta_{3 a_{2}}(s+2 \pi)-\vartheta_{3 a_{2}}(s)=\frac{2 \pi g}{\left[4(j-2 g)\left(g^{2}+1\right)\right]} .
$$

Thus we find that, to this order,

$$
\left|z_{2}\right|=\frac{|g|}{\left[4 i^{2}\left(g^{2}+1\right) \sqrt{\left(4 g^{2}+1\right)}\right]} .
$$

As expected, this is zero if $\beta=0$ (since $g=i \beta$ ).

The above formulae (5.13), (5.17) and (5.22) are compared with numerical results in Figs. 22, 3 14 and 6 respectively. As expected, the perturbation theory is not accurate for the smaller values of $i$, but it agrees quite well with the numerical results for $i>2$.

\section{NUMERICAL RESULTS}

We start with equation (2.2), including real forcing terms:

$$
\beta \theta^{\prime \prime}+\theta^{\prime}+\sin \theta=i+a_{s} \sin \left(\omega^{\prime} \tau\right)+a_{c} \cos \left(\omega^{\prime} \tau\right),
$$

where $a_{s}$ and $a_{c}$ are small (in practise we have verified that, in the range $10^{-4} \leq a_{s}, a_{c} \leq 10^{-3}$, linearity holds within the numerical precision of the calculations), and $\omega^{\prime}$ is adjusted so that

$$
\omega_{J}\left(\omega^{\prime}, a_{s}, a_{c}\right)=\omega^{\prime},
$$

$\omega_{J}$ being the frequency of the solutions of (6.1) (after the decay of transients).

The numerical solution of Eq. (6.1) was investigated using several initial conditions for both $i<1$ and $i>1$. Our main interest here are the cases when $i>1$. In these cases, we start with arbitrary initial conditions at $\tau=0$, and begin measurements on the solution at $\tau=\tau_{0}$ where $\tau_{0}$ is about 100 (when transients have become negligibly small, for all relevant choices of $\beta, i$ and $\left.\omega^{\prime}\right)$. Then, after $N=10^{3}$ cycles, the condition $\theta\left(\tau_{1}\right)-\theta\left(\tau_{0}\right)=2 \pi N$ yields 


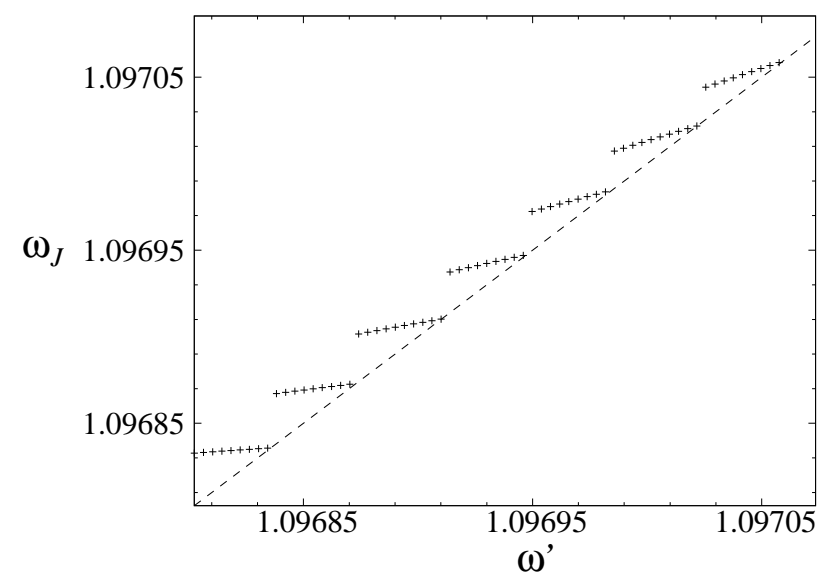

FIG. 1: Graphical representation of the equation $\omega_{J}\left(\omega^{\prime}, a_{s}, 0\right)=\omega^{\prime}$. The crosses are the numerical results for $\omega_{J}\left(\omega^{\prime}, a_{s}, 0\right)$ where $a_{s}=10^{-4}, 2 \times 10^{-4}, \ldots$ and $7 \times 10^{-4}$. In this example $\beta=0.45$ and $i=1.4142$.

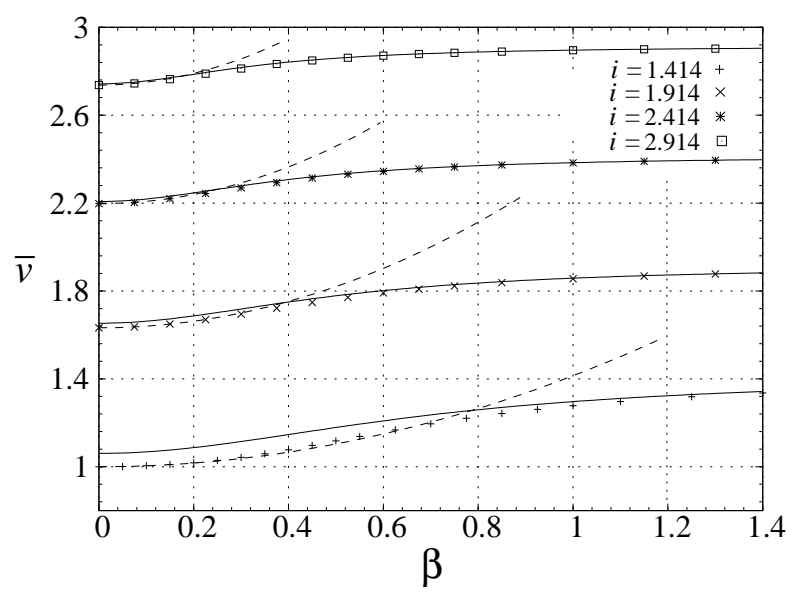

FIG. 2: The dimensionless average voltage as a function of $\beta$ and four values of $i$. The continuous and broken lines are the perturbative results in powers of $1 / i$ and $\beta$ respectively.

the period $\tau_{J}=\left(\tau_{1}-\tau_{0}\right) / N$ (the size of the time step employed in the numerical code was $\Delta \tau=10^{-4}$, between $\tau=0$ and $\tau=\tau_{0}$, and $\Delta \tau=10^{-6}$ after the transients). At the solutions of (6.2), but not otherwise, the results for $\omega_{J}=2 \pi / \tau_{J}$ and $|z|$ are stable against changes in the initial conditions and in $\tau_{0} \simeq 100$ and $N \simeq 10^{3}$.

The next step in the numerical procedure consists in solving (6.2). In the figure 1 we illustrate this procedure with an example of the intersections of $\omega_{J}\left(\omega^{\prime}, a_{s}, 0\right)$ with the diagonal line for $\beta=0.45, i=1.4142$ and a series of values of $a_{s}$. Although figure 1 only shows a few points in the vicinity of the diagonal line, the numerical code employed 40 times more points, in the same range shown in figure 1 in order to reach the required precision in the determination of the impedances. In this range of variation of $a_{s}$, the point of intersection increases linearly with $a_{s}$.

In general the numerical procedure solves (6.2) and

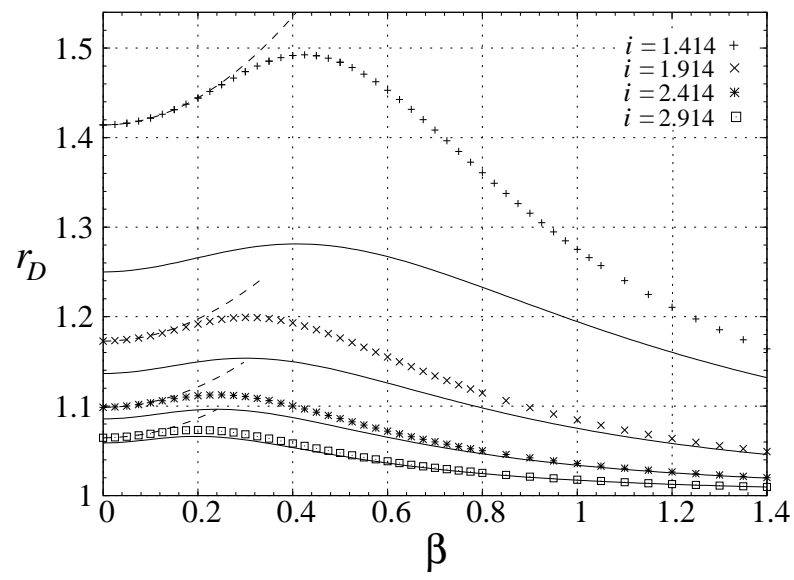

FIG. 3: The dimensionless dynamical resistance as a function of $\beta$ and four values of $i$. The continuous and broken lines are the perturbative results in powers of $1 / i$ and $\beta$ respectively.

\begin{tabular}{|c|c|r|}
\hline$\beta$ & $\frac{\Delta \bar{v}}{\bar{v}} \times 100$ & $\frac{\Delta r_{D}}{r_{D}} \times 100$ \\
\hline \hline 0.00 & 6.066 & 11.611 \\
0.30 & 6.923 & 13.380 \\
0.60 & 4.371 & 12.790 \\
0.90 & 1.921 & 7.760 \\
1.20 & 0.854 & 4.168 \\
1.50 & 0.415 & 2.264 \\
1.80 & 0.221 & 1.289 \\
2.10 & 0.126 & 0.772 \\
2.40 & 0.077 & 0.485 \\
2.70 & 0.049 & 0.318 \\
3.00 & 0.033 & 0.216 \\
3.30 & 0.023 & 0.152 \\
3.60 & 0.016 & 0.109 \\
\hline
\end{tabular}

TABLE I: Differences between the numerical and the $1 / i$ perturbation theory for both $\bar{v}$ and $r_{D}$, for $i=1.41$.

yields the result

$$
\omega_{J}\left(a_{s}, a_{c}\right)=\omega_{J}(0,0)+a_{s} z_{s}+a_{c} z_{c}+\ldots
$$

which gives the Josephson frequency and the two real impedances (for $k=1$ ). (We also have obtained the nonlinear terms in (6.3) which are not important in the present analysis). Using this approach, we were able to perform a detailed numerical calculation of the quantities $\omega_{J}, r_{D},\left|z_{1}\right|=\sqrt{z_{s}^{2}+z_{c}^{2}}$ and $\left|z_{1}\right| / r_{D}$. We also have investigated the higher harmonics $(k=2,3, \ldots)$ and computed the corresponding quantities such as $\left|z_{2}\right|,\left|z_{2}\right| / r_{D}$ and $\left|z_{1}\right|^{2}+2\left|z_{2}\right|^{2}$. Our main interest is to investigate how significant is the dependence on $\beta$ for several values of $i$.

Let us start with the results for $\bar{v}=\omega_{J}(0,0)$ and $r_{D}$ (see Eq. (5.13)). These are shown in figures 2 and 3 as functions of $\beta$ and four values of $i$. From these figures one can see what are the numerical values of $\beta$ and $i$ such that the perturbative results, obtained in the previous 


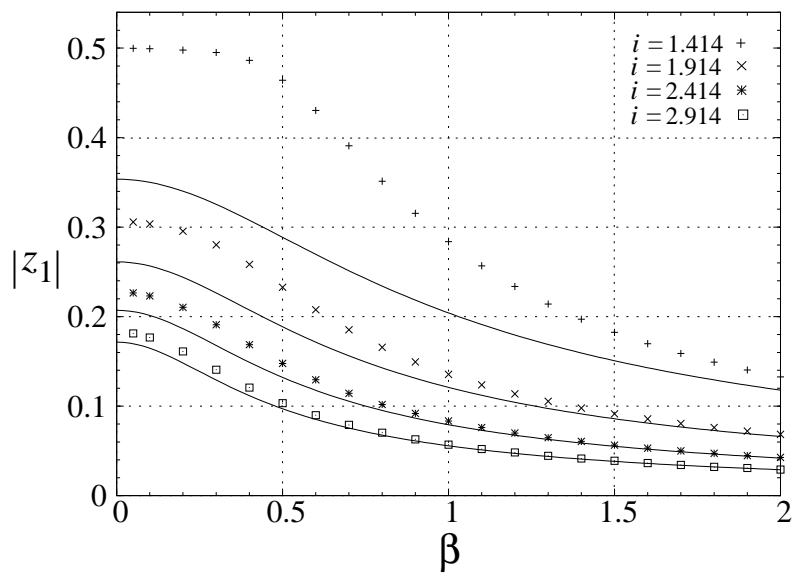

FIG. 4: The impedance associated with the first harmonic as a function of $\beta$ and four values of $i$. The full lines represent the $1 / i$ perturbative result.

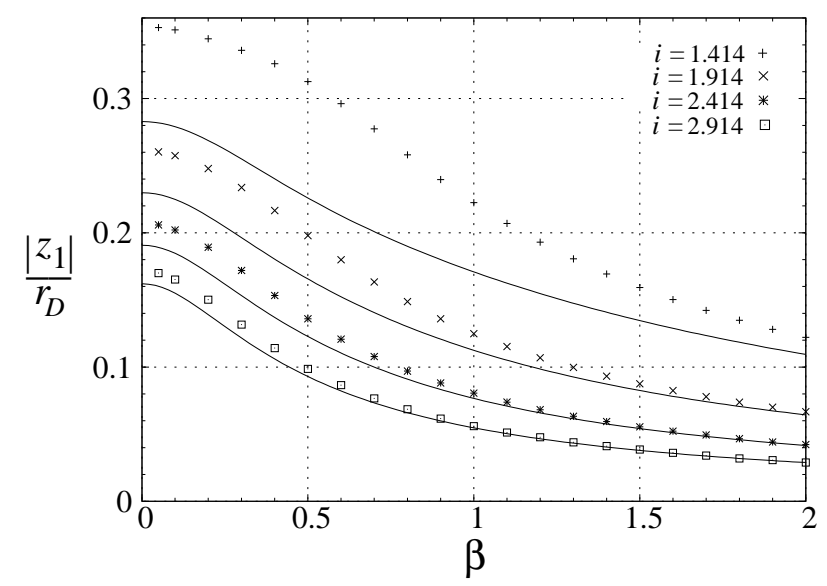

FIG. 5: Same as in figure 4 but divided by $r_{D}$.

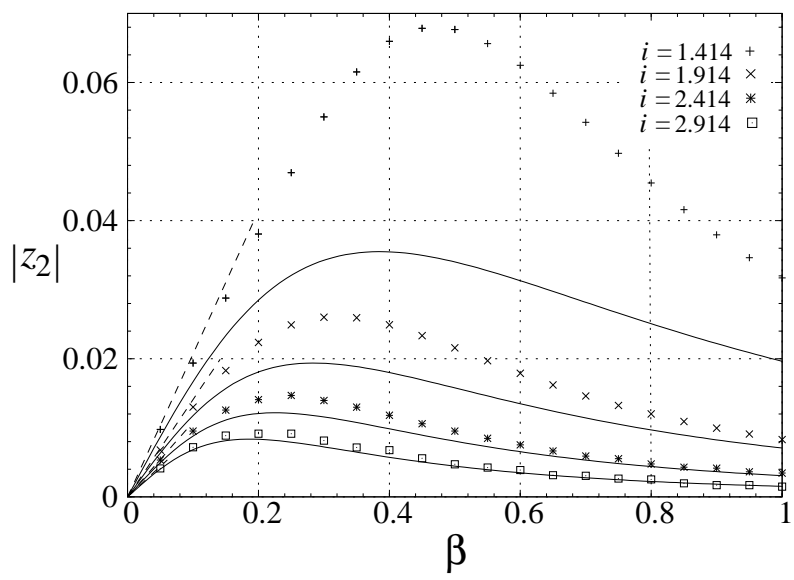

FIG. 6: The impedance associated with the second harmonic as a function of $\beta$ and four values of $i$. The full lines are the $1 / i$ perturbative results and the dashed lines are the order $\beta$ perturbative result given by (4.18).

sections, can be trusted. For instance, the perturbative

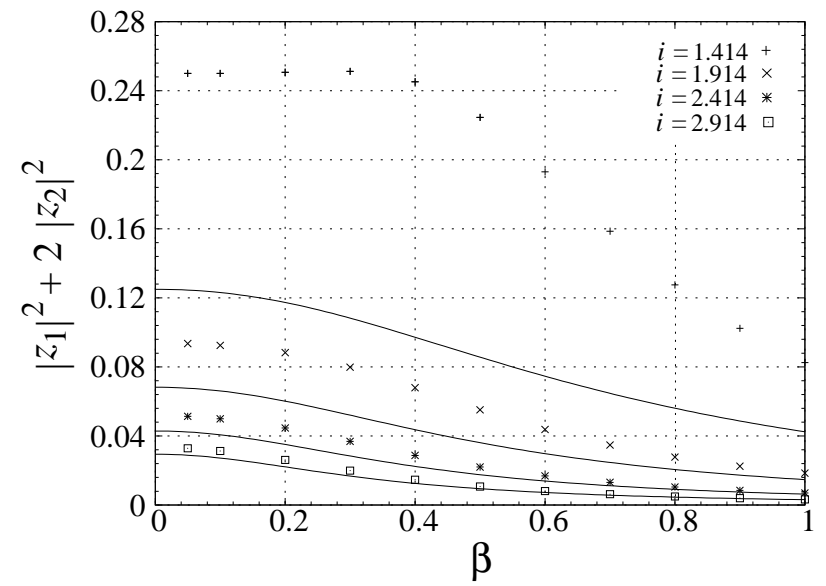

FIG. 7: $\left|z_{1}\right|^{2}+2\left|z_{2}\right|^{2}$ as a function of $\beta$ and four values of $i$. The full lines are the $1 / i$ perturbative results.

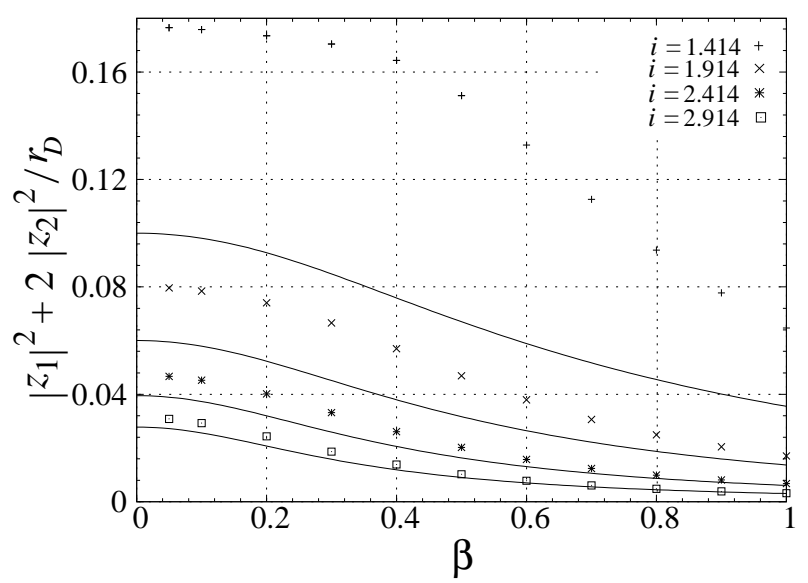

FIG. 8: Same as in figure 7 but divided by $r_{D}$.

increase with $\beta$ only persists up to about $\beta=0.3$, for $i=1.4$; after that $r_{D}$ decreases. Although the numerical computation has been performed up to $\beta=4$, here we are focusing on the interval which may be more realistic from the phenomenological point of view. Besides, larger values of $\beta$ are very well described by the $1 / i$ perturbation theory even for $i=1.4$. For instance, we have found that, for $i=1.4$, the difference between the numerical and the $1 / i$ perturbative result for $r_{D}$ is smaller than $1 \%$ for $\beta>2.0$ (see table 【).

Let us now consider the results for the impedances associated with the forcing terms. The numerical results for $\left|z_{1}\right|=\sqrt{z_{s}^{2}+z_{c}^{2}}$ and $\left|z_{1}\right| / r_{D}$ are shown in figures 4 and 5. respectively. Similarly to the case of the dynamical resistance the curves for the $1 / i$ perturbative result (full lines) underestimate the exact numerical points by an amount which becomes negligible when $i$ increases. Also, the perturbative result becomes indistinguishable from the exact result for $\beta$ sufficiently large.

The results for the impedances associated with second harmonic are shown in figure 6 The validity of the per- 


\begin{tabular}{|c|c|c|}
\hline$i$ & $r_{1}$ & $r_{2}$ \\
\hline \hline 1.41 & 0.96 & 0.90 \\
1.91 & 0.75 & 0.72 \\
2.41 & 0.55 & 0.56 \\
2.91 & 0.44 & 0.49 \\
\hline
\end{tabular}

TABLE II: The ratios $r_{1} \equiv\left[Z_{1}(\beta=0.38) / Z_{1}(\beta=0)\right]^{2}$ and $r_{2} \equiv\left[\left(Z_{1} / R_{D}\right)(\beta=0.38) /\left(Z_{1} / R_{D}\right)(\beta=0)\right]^{2}$ are shown in columns 2 and 3 , respectively, for the values of $i$, shown in column 1. Notice that these ratios are the same if we use either $Z_{1}$ and $R_{D}$ or $z_{1}$ and $r_{D}$.

turbative results for small $\beta$ or large $i$ can be seem in the figure. As in the previous cases the curves for the $1 / i$ perturbative result become very close to the exact numerical result for $\beta$ sufficiently large. Finally, in figures 7 and 8 we shown the results for $\left|z_{1}\right|^{2}+2\left|z_{2}\right|^{2}$ and $\left(\left|z_{1}\right|^{2}+2\left|z_{2}\right|^{2}\right) / r_{D}$. These quantities are relevant for the calculation of the low frequency voltage noise (according to the Eqs. (2.10) and (4.19) ).

\section{CONCLUSIONS}

We have obtained the generalized impedances $Z_{k}$ for a range of values of the two dimensionless parameters $\beta$ and $i$. These allow one to find the current noise strength from (2.10). We are particularly concerned with the dependence on the parameter $\beta$ which measures the importance of the capacitance of the junction. For $\beta=0$ the Langevin equation (1.4) has a simple analytic solution. An important question is how far the results for $\beta=0$ provide a good approximation when $\beta$ is of order 0.5 . Figures 2 to 8 and table $\amalg$ exhibit the dependence on $\beta$, which leads to significant corrections to the voltage noise.

Since the analysis used in [1] neglected the $\beta$ dependence [13] the conclusions of that paper may not be as decisive as claimed. If similar experiments are done in the future, our results should be useful.

\section{Acknowledgements}

F.T.B. and J.F. would like to thank CNPq, Brazil, for a grant. 
[1] R. H. Koch, D. J. Van Harlingen and J. Clarke, Phys. Rev. B26, 74 (1982).

[2] U. Gavish, Y. Levinson and Y. Imry, Phys. Rev. B 62, R10637 (2000).

[3] C. Beck and M. C. Mackey, Phys. Lett. B605, 295 (2005); Physica A379, 101 (2007).

[4] J. C. Taylor, J. Phys. Condens. Matter 19 19, 106223 (2007).

[5] G. W. Ford, J. T. Lewis and R. F. O'Connell, Phys. Rev. A37, 4419 (1988).

[6] P. Coullet, J. M. Gilli, M. Monticelli and N. Vandenberghe, American Journal of Physics 73, 1122 (2005).

[7] R. H. Koch, D. J. Van Harlingen and J. Clarke, Phys. Rev. Lett. 45, 2132 (1980).

[8] H. B. Callen and T. A. Welton, Phys. Rev. 83, 34 (1951).
[9] V. Branchina, M. Di Liberto and I. Lodato, Journal of Cosmology and Astroparticle Physics 0908, 011 (2009).

[10] Y. Levinson, Phys. Rev. B 67, 184504 (2003).

[11] R. F. Voss, Journal of Low Temperature Physics 42, 151 (1981).

[12] L. Ponta, A. Carbone, M. Gilli and P. Mazzeti, Phys. Rev. B 79, 134513 (2009).

[13] In [1] it is actually assumed that equation (2.13) is a good approximation with observed values of $R_{D}$ and $\bar{V}$, whereas we have used the theoretical values. From Fig. 3 of [1], it seems that the measured value of $R_{D}$ in the important range is consistent with the theoretical value to within about $10 \%$, but from the data in the published paper we cannot be more precise about this. 